American Journal of Applied Sciences 6 (2): 247-250, 2009

ISSN 1546-9239

(C) 2009 Science Publications

\title{
Visualization of Available Power Transfer Capability in a Transmission System Using Morphological Techniques
}

\author{
S.U. Prabha, B.S. Dayasagar and C. Venkataseshaiah \\ Faculty of Engineering and Technology, \\ Multimedia University, Melaka Campus, 75450 Melaka, Malaysia
}

\begin{abstract}
A morphological decimation technique has been proposed and implemented to analyze the available power transfer capability in a transmission power network. The method creates a graphical image of the power network with thickness of the lines proportional to their respective rated megavolt ampere (MVA) capacity. Based on ac load flow solution, another image was created to represent the power flow in Megawatt (MW) between the buses. Proper scaling procedure has been discussed for the construction of graphical images. The novelty of this research lies in the application of mathematical morphological techniques for decimating the created images. The image created for the MW capacities of the power lines were decimated into categories and grouped into different colors for better visualization. The multi-color image is superimposed on the input image which is created for the MVA capacity of the network. The proposed method has been tested on an IEEE test system. The results from the present approach can help the planner and operator in a power station, to get a better visualization of the power network. This is the first time this kind of multi-color visualization is presented and it can be used to find the optimal path for power transfer from one bus to another.
\end{abstract}

Key words: Image processing, visualization, power system planning, power system monitoring

\section{INTRODUCTION}

The electrical power systems are very large and complicated networks. Power system operators need to interpret and integrate multiple measured parameters. Power availability and its utilization are the two significant parameters that are usually denoted by numerical values in MVA and MW units. Policy and decision makers consider these parameters to propose appropriate planning schedules to take care of the further power demands from the point of transferability from one zone to another. Analysis based on these numerically denoted parameters requires both expertise and understanding of statistics to properly summarize the power utilization and demands. If such statistical summaries can be represented as spatial phenomena over a geographic space, one can have a synoptic view of the total power availability and its utilization between the buses with significant contrast. This is a kind of visualization of the available maximum power capacity and the utilized capacity in terms of spatial maps.

This study addressed in this research with associated analysis is carried out based on certain map algebraic tools like mathematical morphology. In earlier studies, various methods for load flow tracing have been reported ${ }^{[1]}$. A morphological image analysis technique was presented to visualize the strong sub networks in a power system ${ }^{[2]}$. Different schemes of network representation using various aspects of transmission system were briefly discussed $\mathrm{in}^{[2]}$. The purpose of the present work is to use the morphological decimation techniques to visualize the available power transfer capability in a transmission system. The special features of this proposed approach are multi-color visualization of the available power transfer capability in a transmission network and its application to optimal path finding for power transfer between any two buses.

\section{MATERIALS AND METHODS}

The IEEE 24 bus test system is considered as an example. Figure 1 shows a single-line diagram of the power network under consideration. The thickness of the line represents the rated megavolt-ampere (MVA) capacity. This is referred as input image. Using the results of power-flow analysis ${ }^{[4]}$, another image is constructed for the megawatt (MW) flow in the lines and is shown in Fig. 2. These images were translated into eight-bit bitmap images. The image constructed

Corresponding Author: S.U. Prabha, Lecturer, Faculty of Engineering and Technology, Multimedia University, Melaka Campus, 75450 Melaka, Malaysia Tel: +606 2523954 Fax: +606-231 6552 


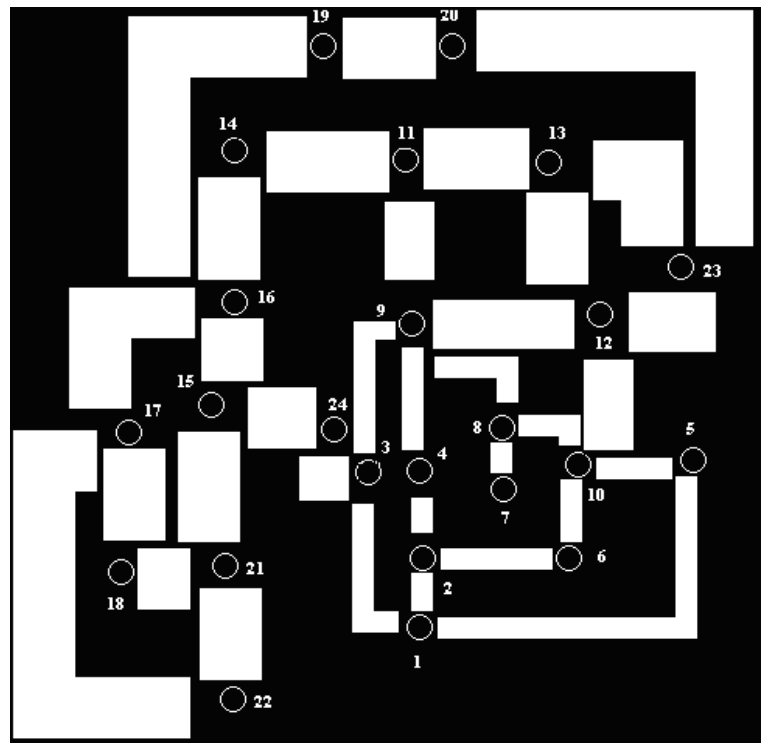

Fig. 1: Image created with MVA capacities

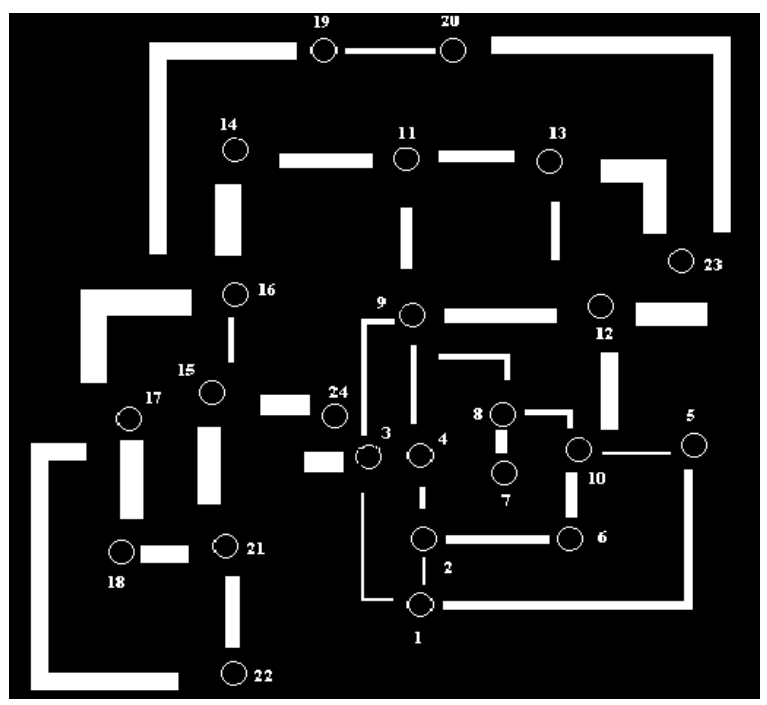

Fig. 2: Image created with MW capacities

with MW flow in the lines is decimated through a multiscale morphological opening transformation ${ }^{[3]}$, such that the decimated image is represented as 9category power flow network. This processed image is then superimposed on the input image. The comparison could be done between the used capacity and the maximum capacity to obtain the index of availability.

Image construction techniques: The logic in creating the input image in terms of pixels with varied ranges is based on the following steps.
Step 1: The largest capacity of the line is 500 MVA. The other input MVA capacities are 175 and 400. The later capacities are divided with the largest possible capacity to normalize all the MVAs. Accordingly the capacity values are respectively $0.35,0.8$ and 1.0 .

Step 2: In order to make sure that the amount of MW that is flowing between the buses, which will always be the subset of the maximum allowable MVA capacity, the ratios obtained at step 1 are considered as the basis to decide the width of the input MVA in terms of pixels.

Step 3: Assigning pixels by taking the condition cited in step 2 yields the pixel width 14, 32 and 40 which clearly acts as sets of the subsets within the ranges of 2-8, 10-12 and 14-20.

The image created for the MW flow between the buses is also drawn to scale. The number of pixels corresponding to the thickness of each line is chosen after grouping the line flow values, i.e., 0 to $20 \mathrm{MW}$ flow capacity is represented by the thickness of 2 pixels and henceforth the proportionality is maintained. The images that are constructed by the process explained above yield a bitmap image of the power grid as shown in Fig. 1 and 2. It would be appropriate if these spatially distributed diagrams are decimated according to their potentialities.

Morphological transformations: The required basic transformations are briefly explained as follows. Let A, $\mathrm{P}$ and $\mathrm{M}$ denote sets representing total available power capacity, buses (open circles) and the power being utilized respectively over two-dimensional discrete space on black background. These sets depicting the important features in spatial form are created interactively with white pixels and black background. Figure 1 is obtained through logical union of sets A and P. It is also obvious from Fig. 1 and 2 that the set M, being the map denoting spatial distribution of the load being utilized, is a subset of set A. Morphologic transformations are explained with an image represented in discrete space (M) and a template (B) that would be used as a probing rule to make modifications in $\mathrm{M}$. The basic binary morphologic transformations include erosion and dilation. Figure 3 shows the impact of the basic morphological transformations.

Erosion: $\mathrm{M} \ominus \mathrm{B}$

Dilation: $\mathrm{M} \oplus \mathrm{B}$ 
Am. J. Applied Sci., 6 (2): 247-250, 2009

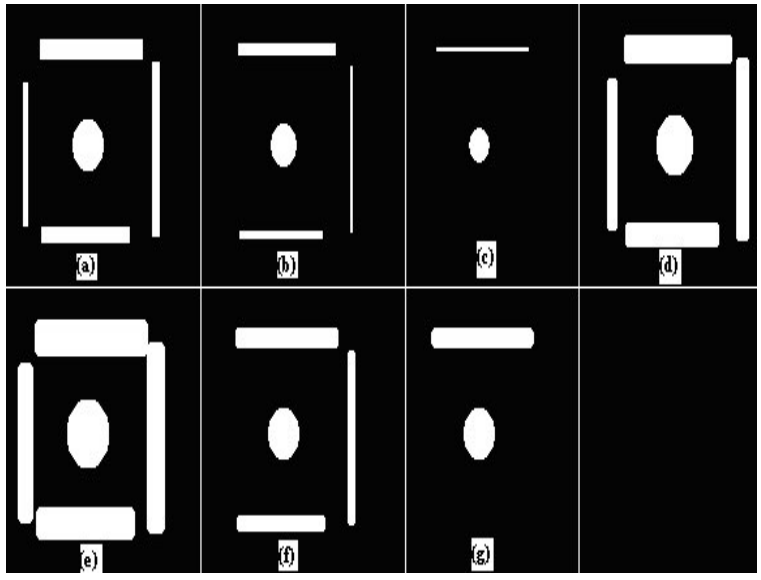

Fig. 3: (a): Synthetic power network (white) and nonetwork zone (black) indicating the network segment of various widths; (b): Network after performing one cycle of erosion; (c): After two cycles of erosion; (d): After one cycle of dilation; (e): After two cycles of dilation; (f): After one cycle of opening and (g): After two cycles of opening

$$
\text { Opening: } \mathrm{M} \ominus \mathrm{B} \oplus \mathrm{B}=\mathrm{MoB}
$$

where, $\ominus$ and $\oplus$ respectively denote erosion and dilation symbols and $\mathrm{B}$ denotes a symmetric square structuring element of primitive size $3 \times 3$. Opening transformation is performed by performing erosion transformation followed by dilation transformation on $\mathrm{M}$ with respect to $\mathrm{B}$.

Decimation of set $M$ via multi-scale opening: The binary set depicting the power network in spatial form is further subjected to decimation process in the following steps.

Step 1: Decimation of into varied categories.

Step 2: Color coding of category-wise decimated network.

Step 3: Union of color-coded network segments to visualize the network as a function.

The whole aim of the above step-wise procedure is to depict the power-network as a spatially represented function. This function further facilitates ways to explore links with proper power planning tasks. In this approach, decimation of set is done via multiscale opening transformation as per Eq. 4:

$$
\begin{aligned}
& (\mathrm{MoB})=(\mathrm{MoB}) \\
& (\mathrm{MoB}) \mathrm{oB}=(\mathrm{Mo} 2 \mathrm{~B}) \\
& \cdot \\
& \underbrace{[(\mathrm{MoB}) \mathrm{oB}] \mathrm{oB} \ldots \mathrm{oB}]}_{n \text {-times }}=(\mathrm{MonB}) \\
& [(\mathrm{n}) \mathrm{B}] \text { is }\rangle(\mathrm{n}-1) \mathrm{B} \text { or }(\mathrm{n}-1) \mathrm{B}\langle[(\mathrm{nB})]
\end{aligned}
$$

Multiscale opening is performed on set $\mathrm{M}$ that consists of power lines of various thicknesses. In this set, different thickness imply different MW capacities of the lines connecting the buses. This synoptically and spatially represented set $M$ is subjected to opening transformation by means of increasing sizes of structuring elements (B) with an aim to distribute power grid lines according to their widths. A set of equations explored in the distribution process are as follows.

Further to precisely isolate the power grid lines of specific width in an increasing order, we subtract each degree of opened version from opened version of previous degree of opening (which is lower level of opening). This process is mathematically shown as follows:

$$
\left(\mathrm{GS}_{\mathrm{n}}\right)=[(\mathrm{Mo}(\mathrm{n}-1) \mathrm{B}) /(\mathrm{MonB})]
$$

where, $[(\mathrm{M} \ominus \mathrm{nB} \oplus \mathrm{nB}]=(\mathrm{MnoB})$ and $(\mathrm{M} \ominus \mathrm{nB}) \neq \phi$, $(\mathrm{M} \ominus(\mathrm{n}+1) \mathrm{B}) \neq \phi$ and $\mathrm{GS}_{\mathrm{n}}$ denotes $\mathrm{n}^{\text {th }}$ category power grid segment. In Eq. 5 the reverse solidus denotes subtraction process between spatially represented power network sets. Implementing this equation, the opened versions of $\mathrm{M}$ obtained with recursive approach are considered to isolate width-wise power grid lines.

Color-coded morphological transformation: The proposed scheme for color coding of the decimated set (M) is presented as follows. For better visualization in a single image, each isolated power grid line of $\mathrm{n}^{\text {th }}$ category $(\mathrm{n}=1,2,3 \ldots \mathrm{N})$ is color-coded with intensity value denoted by (i), where $\mathrm{n}=\mathrm{i}$. Each $n$ th-category grid-segment $(\mathrm{n}=[1,2, \ldots \mathrm{N}])$ decimated for 24-bus system is color-coded as follows Eq. 6:

$$
\bigcup_{\substack{n=0 \\ \mathrm{i}=n+1}}^{\stackrel{9}{N}}\left[\mathrm{GS}_{\mathrm{n}}\right]^{\mathrm{i}}
$$

where, $\mathrm{N}$ is the maximum number of segments that could be decimated and i denotes the color employed to assign $\mathrm{n}^{\text {th }}$ degree grid segment. On processing the bitmap image shown in Fig. 2, the color-coded image is 
obtained by using the proposed scheme. The nine categories are obvious with different colors. This decimated color-coded image is super imposed on the input image as shown in Fig. 4.

\section{RESULTS AND DISCUSSION}

The IEEE 24 bus test system was considered to demonstrate the proposed scheme. Different structuring elements like square, octagon and rhombus have been tried to test the algorithm. The octagon shaped structuring element gave the best results and hence this has been used in the present work. The difference between the MVA (in white color) and MW (in nonwhite color) gives the amount of available power that is unused. The ratio of MVA capacity to MW capacity of the line connected between two buses can be used as an index of availability. Considering the test system as one zone, the power transfer between any two buses can be established by identifying the possible paths and choosing the optimal paths between a pair of buses.

In the system under consideration, by visualizing Fig. 4, seven possible paths for the power transfer between bus 13 (slack bus) and bus 18 (load bus) were identified and presented in Table 1. The paths 1, 2, 3 and 4 allow less amount of power transfer. Path 5 is not

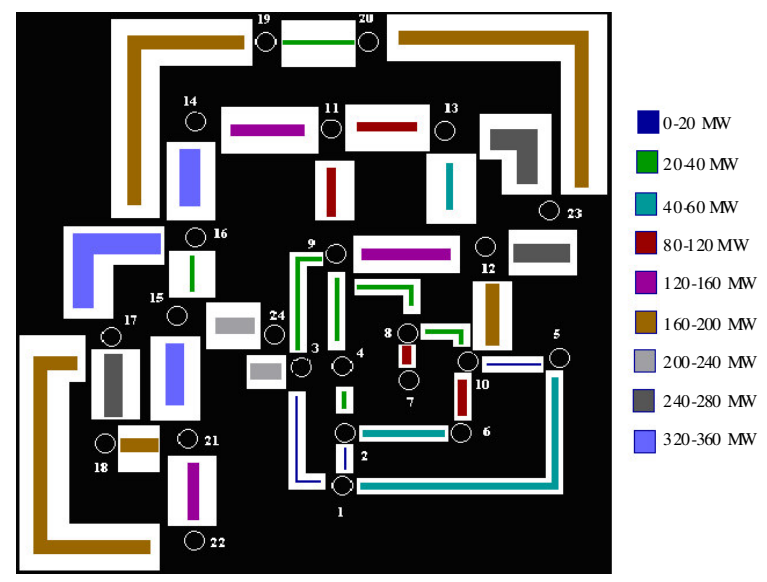

Fig. 4: Decimated color-coded image superimposed on the input image

Table 1: Paths for power transfer

\begin{tabular}{ll}
\hline S. No. & Paths \\
\hline 1 & $13-11-14-16-17-18$ \\
2 & $13-11-14-16-15-21-18$ \\
3 & $13-23-20-19-16-17-18$ \\
4 & $13-23-20-19-16-15-21-18$ \\
5 & $13-12-9-3-24-15-21-18$ \\
6 & $13-11-14-16-17-22-21-18$ \\
7 & $13-12-23-20-19-16-17-22-21-18$ \\
\hline
\end{tabular}

suitable for power transfer because of the fact that the available MVA capacity between buses 9 and 3 is only 175, which does not allow more power to be transferred. Paths 6 and 7 can be considered for more power transfer. Out of all the above, path 7 provides the optimal path.

\section{CONCLUSION}

The proposed technique was successfully implemented on IEEE 24 bus test system and the results obtained were found to be satisfactory. The contributions can be summarized as follows:

- A scheme to generate a graphical image of a power network to scale, for a better visualization in twodimensional discrete space has been developed

- A method based on morphologic filtering to decimate the power network according to their potentials has been proposed and demonstrated

- Further, optimal path finding procedure for power transfer between two buses has been presented

These can be extended to find optimal path for available power transfer between two areas/zones in a larger power network. The proposed techniques could be used by the planners and operators to visualize the network and extract the abstract information of the transmission system easily. It is envisaged that the proposed scheme finds wider application in power system planning and monitoring.

\section{REFERENCES}

1. Wu, F.F., Y. Ni and P. Wei, 2000. Power transfer allocation for open access using graph theoryfundamentals and applications in systems without loop flow. IEEE Trans. Power Syst., 15: 923-929. Doi: S 0885-8950(00)07723-3.

2. Radhakrishnan, P., B.S.D. Sagar and B. Venkatesh, 2005. Morphological image analysis of transmission systems. IEEE Trans. Power Delivery, 20: 219-223. Doi: 10.1109/TPWRD. 2004.839213.

3. Serra, J., 1982. Image Analysis and Mathematical Morphology. Academic Press, London.

4. Umapathy, P. and C. Venkataseshaiah, 2007. A novel approach for the assessment of available transfer capability in power systems. College Sci. India, 1: 1-4. http://www.collegescienceinindia. com/dec2007/index.html. 